\title{
Den Durchblick behalten
}

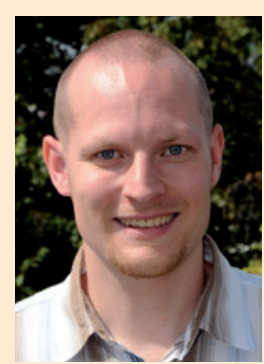

Christian Schäfer, Stuttgart
Was ist bei der alltäglichen Behandlung von Patienten eine der tragenden Säulen für Ärzte und auch für Pflegekräfte? Genau, es ist die Kenntnis der aktuellen Studienlage, dem State of the Art in einer Disziplin und den gültigen Leitlinien. In der Nephrologie sind relativ viele Leitlinien und Studien zu berücksichtigen. Das liegt u.a. daran, dass die Niere eng und stark mit anderen Organsystemen im Körper verflochten ist und in vielen Ausprägungsformen einen großen Einfluss auf den Gesundheitszustand der Patienten hat. Man denke neben der Entgiftungsfunktion bzw. Harnproduktion der Niere beispielsweise an ihre Wechselwirkungen mit dem Knochen- und Mineralhaushalt, dem Hormonhaushalt bzw. dem Eisenstoffwechsel und kardiovaskulären Eigenschaften, zu denen auch die Verkalkung der Gefäße und der Blutdruck gehören.

In diesem Zusammenhang war der Welt-Hypertonie-Tag am 17. Mai sicherlich eine hilfreiche Veranstaltung zur Aufklärung der Bevölkerung: Menschen wurden mit dem Motto „Bluthochdruck erkennen und behandeln“ dafür sensibilisiert, regelmäßig ihren Blutdruck zu kontrollieren und einen Hypertonus therapieren zu lassen. Denn ein langjähriger Bluthochdruck kann zu einem chronischen Nierenversagen, einem Herzinfarkt und/ oder einem Schlaganfall führen. Laut Deutsche Hochdruckliga e. V. (DHL) leben in Deutschland 20-30 Millionen Menschen mit Hypertonie, aber nur etwa die Hälfte weiß von dem „silent killer“, der sie begleitet - diese Informationen können daher Leben retten.

Dem Bluthochdruck bei Nierenkranken haben wir dieses Schwerpunktheft gewidmet: In den letzten 1,5Jahren seit dem Erscheinen der letzten Ausgabe der Dialyse aktuell zum Thema Hypertonie hat sich bzgl. neuer Leitlinien auf diesem Gebiet sehr viel getan. Somit tragen die interessanten Beiträge ab Seite 250 dazu bei, dass Sie, liebe Leser, sich auf den aktuellen Stand bringen können bzw. den Durchblick behalten, was die Essenz zur Blutdruckeinstellung bei Niereninsuffizienz ist. Gerne können Sie Ihr Wissen nach der Lektüre der Artikel überprüfen, und zwar mithilfe des CME-Fragebogens im Anschluss an den Schwerpunktteil. Haben Sie als Arzt mindestens $70 \%$ der Fragen richtig beantwortet, erhalten Sie hierfür wie gehabt 3 CME-Fortbildungspunkte. Wie schon oben angemerkt, ist es nicht nur für Nephrologen wichtig, sich über den Wissensstand in ihrem Fachgebiet zu informieren. Auch Pflegekräfte sollten die für sie relevanten aktuellen Studien und Leitlinien kennen. Um Ihnen den Erwerb des pflegewissenschaftlichen Wissens zu erleichtern, haben wir ab dieser Ausgabe der Dialyse aktuell die Rubrik „Journal-Club Pflege“ eingeführt (ab S. 248). Dr. rer. medic. Dietmar Wiederhold, Heilbad Heiligenstadt, stellt Ihnen ab jetzt voraussichtlich in allen ungeraden Ausgabennummern relevante Studien aus der nephrologischen Pflege vor.

Die Pionierin der modernen westlichen Krankenpflege Florence Nightingale (1820-1910) würde es sicherlich begrüßen, wenn sie mitbekäme, dass in der nephrologischen Pflege eine evidenzbasierte und studienabgesicherte Arbeit mehr und mehr Einzug hält. Nightingales Geburtstag feierte man mit dem Internationalen Tag der Pflegenden am 12. Mai mit zahlreichen Aktionen. In diesem Zusammenhang sagte der Bevollmächtigte der Bundesregierung für Patienten und Pflege Karl-Josef Laumann (CDU), dass sich die Wertschätzung für Pflegekräfte v.a. auch in einer angemessenen Vergütung zeigen muss. Dies ist sicherlich eine passende Einschätzung der Lage, der hoffentlich bald Taten folgen. In diesem Sinne wünsche ich Ihnen eine erkenntnisreiche Lektüre! 